\title{
Effect of Colic Vein Ligature in Rats with Loperamide-Induced Constipation
}

\author{
Flavia Neri, ${ }^{1,2}$ Giuseppe Cavallari,, ${ }^{1,2}$ Matvey Tsivian, ${ }^{1,2}$ Elisa Bianchi, ${ }^{1,2}$ Rita Aldini, ${ }^{2}$ \\ Monica Cevenini, ${ }^{2}$ Elena Guidetti, ${ }^{2}$ Gian Luca Piras, ${ }^{2}$ Milena Pariali, ${ }^{2}$ and Bruno Nardo ${ }^{1,2}$ \\ ${ }^{1}$ Department of General Surgery and Transplantation, S. Orsola-Malpighi Hospital, University of Bologna, 40126 Bologna, Italy \\ ${ }^{2}$ Center for Applied Biomedical Research (CRBA), S. Orsola-Malpighi Hospital, University of Bologna, 40126 Bologna, Italy
}

Correspondence should be addressed to Giuseppe Cavallari, giuseppe.cavallari3@unibo.it

Received 29 November 2011; Revised 6 February 2012; Accepted 20 February 2012

Academic Editor: Monica Fedele

Copyright () 2012 Flavia Neri et al. This is an open access article distributed under the Creative Commons Attribution License, which permits unrestricted use, distribution, and reproduction in any medium, provided the original work is properly cited.

\begin{abstract}
Introduction. Medical treatment in chronic constipation is not always successful. Surgery is indicated in unresponsive selected severe cases. This study presents the distal venous colic ligation in rat as a novel surgical approach. Materials and Methods. 16 rats (study group) were evaluated in 3 phases of 6 days each: A (normal conditions), B (loperamide-induced constipation), and C (colic vein legation) and compared with rats treated in phase C with PEG 4,000 (control group). Blood biochemical and physiological parameters, daily fecal water content (FWC), and histological analysis were performed in all study phases. Results. No biochemical and physiological parameters changes were observed. FWC decreased in phase B and increased in phase $\mathrm{C}$ in both groups with a grow up to 2.3 -fold in study group compared to control $(P<0.0001)$. Moreover, in study group, a high number of colonic goblet cells were detected (phase $\mathrm{C}$ versus phase B: $P<0.001$ ) while no differences were registered in control. Conclusion. By ligature of the colic vein in constipated rats, an increase in FWC and goblet cells higher than in PEG treated rats was detected. The described surgical procedure appeared effective, simple, and safe; further studies in animal models, however, are necessary to assess its clinical applicability.
\end{abstract}

\section{Introduction}

A consensus definition of constipation was reached in the ROME conference held in 1992 with a further revision in 2001 [1]. Two or more of the following symptoms, present in the previous 3 months, were considered diagnostic for constipation: symptom onset at least 6 months before diagnosis; straining during at least $25 \%$ of defecations; lumpy or hard stools in at least $25 \%$ of defecations; sensation of incomplete evacuation for at least $25 \%$ of defecations; sensation of anorectal obstruction/blockage for at least $25 \%$ of defecations; manual maneuvers to facilitate at least $25 \%$ of defecations; fewer than 3 defecations/week [1].

For its high prevalence constipation represents also an extensive economical burden in terms of general and specialistical medical care due to diagnostic procedures, hospitalization, and therapy $[2,3]$. The pathogenesis of chronic constipation is often unclear; in only about $10 \%$ of cases some metabolic or structural etiological conditions are identified [4]. Generally, each patient's complaint is different, making it difficult to understand the problem and find appropriate therapeutic measures. Lifestyle changes are not often successful, and different types of laxatives are usually necessary. Pharmacological treatment is the first therapeutical choice for constipation and surgery is only reserved as the last option when conservative treatments have failed, and therefore it should be limited to refractory constipation, in most cases due to mixed conditions [1]. Colon resection, in particular, seems to have beneficial effects in some cases of inertia coli [5]. Although the great effort in identifying the best treatment options for patients affected by chronic constipation, the results are often still partial and unsatisfactory [1-6].

In the attempt to address this issue we took inspiration from our direct clinical experience [7]. In that occasion we treated a 25-year-old women with acute liver failure for intoxication from tetracycline, through the arterialization of the portal vein. In particular, with the aim to increase the 
oxygenation of the blood coming to the liver through the portal system, a branch of the inferior mesenteric artery was connected to the inferior mesenteric vein. At longterm follow-up, the patient referred a reversal of the chronic constipation that she had always experienced before. We hypothesized that the physiopathological mechanism for the resolution of constipation was that the ligation of the inferior mesenteric vein, performed to allow the anastomosis between the arterial and the venous splanchnic system, by impairing the colic vein drainage, must decrease the reabsorption of the luminal solute and water, increasing the colonic water content.

Loperamide is a synthetic opioid agonist, able to induce a constipation in animal model by extension of the evacuation time [8], inhibition of colonic peristalsis [9], and reduction of colonic mucus [10]. In order to better characterize the correlation between colic vein congestion and improved fecal transit, the aim of this study was to evaluate the effect of the colic vein ligature in rat with loperamide-induced constipation.

\section{Materials and Methods}

2.1. Animals and Constipation Induction. In our study we used 25 male Sprague-Dawley rats (Charles River Laboratories, Calco, Italy) weighing about $400 \mathrm{~g} /$ b.w.: 16 rats were enrolled in study group and 9 in control group. All animal procedures were approved by the local committee for care and use of laboratory animals of the University of Bologna and performed according to Italian governmental and international guidelines on animal experimentation. Constipation in rats was induced by injection of loperamide (Sigma-Aldrich) at the dose of $0.15 \mathrm{mg} / 100 \mathrm{~g}$ twice a day subcutaneously.

2.2. Study Design. The animals were subjected to a three study phases. The first phase (A; from day 1 to 6 ) was the control period, useful to collect the basal physical parameters of each animal. The second phase (B; from day 6 to 12 ) was the period of loperamide-induced constipation. In the third phase ( $\mathrm{C}$; from day 12 to 18 ) loperamide was continued and animals of study group underwent colic vein ligation (Figure 1), while animals of control group were administered PEG 4,000 (Sigma-Aldrich) ( $250 \mathrm{mg} / \mathrm{kg} /$ day). The equivalent dose for the drug to be administered to rat could not be calculated by the usual formula corrected for the ratio of human serum albumin $\mathrm{Kg} / \mathrm{rat}$ serum albumin $\mathrm{Kg}$ [11], due to the very poor intestinal absorption of PEG 4,000 (from 0.05 and $2.5 \%$ ). Since this formula cannot apply to drugs with little or no intestinal absorption, we chose a dose which was $50 \%$ of the minimal dose inducing toxic effects, such as severe diarrhoea and tremor, obtained by the previous evaluation of the clinical effect of the drug in a dose-response curve from $100 \mathrm{mg} / \mathrm{kg} / \mathrm{day}$ to $1000 \mathrm{mg} / \mathrm{kg} /$ day in a cohort of healthy rats (data non reported). To perform a histological comparative analysis of the colic mucosa in the different study phases, 4 of the 16 animals used were sacrificed at the end of phase A, 4 at the end of phase B, and 8 at the end of phase C. Three animals of the control group were

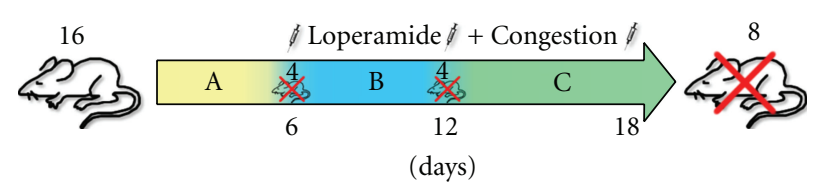

Figure 1: Study group design.

similarly sacrificed at the end of each phase. Biochemical, physiological, and histological parameters of the animals were evaluated through the three sequential phases, for a total of 18 days.

\subsection{Biochemical and Physiological Parameters. We assessed} serum osmolarity and concentration of proteins, sodium, and chloride. The analysis was performed at the end of the three study phases (A, B, and C).

The physiological parameters daily evaluated in our study were body weight, food and water intake, urine volume, weight of wet, and dry fecal pellets. These parameters were collected every morning at the same time in order to minimize the physiological variables. The procedure of feces drying was performed with UNIVAPOR vacuum concentrator. The fecal samples were let to dry for 24 hours at room temperature obtaining a complete removal of water and other volatile substances. The water content of the fecal pellet was calculated as the difference between the wet and dry weights of the pellet.

2.4. Histology. The colic mucosa of all the animals was examined using periodic acid-Shiff (PAS) staining to detect the presence of the intracellular mucus, identifying the number of goblet cells. For this experiment a standard PAS staining protocol was adopted; after deparaffinization and hydratation to water, the slides were oxidized in $0.5 \%$ periodic acid solution for 5 minutes, then rinsed in distilled water and placed in Schiff reagent for 15 minutes. Afterwards the slides were washed under tap water for 5 minutes and then counterstained in Mayer's hematoxylin for 1 minute before the dehydration and the placement of the mounting medium and coverslip. The slides were then analyzed under an optical microscope, and the number of PAS positive cells in the crypts of colic epithelium among the different groups was counted: three fields per slide at a magnification of $20 \mathrm{X}$, three slides per animal.

2.5. Surgical Procedure. The animals were anesthetized through intraperitoneal injection of xylazine-tiletaminezolazepam (Zoletil, Virbac) at the dose of $0.1 \mathrm{~mL} / 100 \mathrm{~g}$. A median xipho-pubic incision was performed in order to expose the abdominal organs and the intestinal vessels. The colic vein was identified at the inferior edge of the pancreas and ligated and cut under microscope visualization (Figure 2). After the procedure an immediate colic venous congestion was observed below the ligation. The abdominal wall was then closed in two layers. 


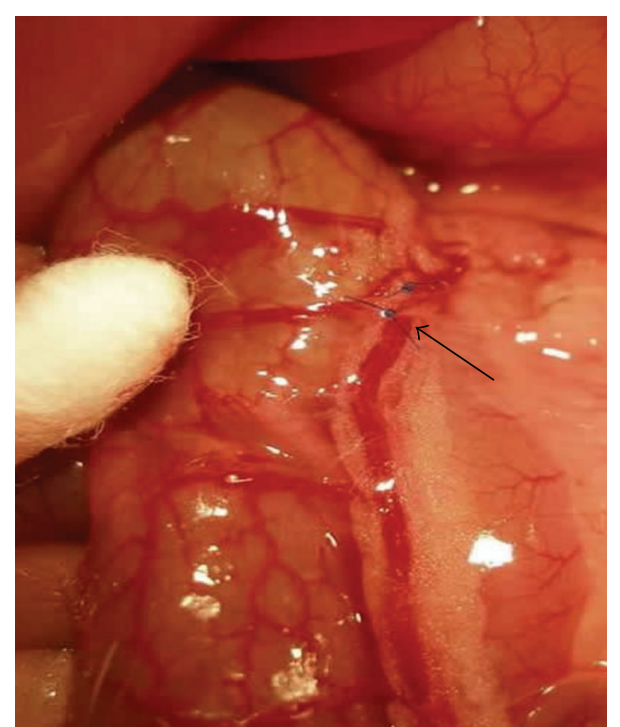

FIgURE 2: Ligation and cut of the colic vein.

\section{Results}

\subsection{Biochemical and Physiological Parameters}

Study Group. No significant differences of the biochemical parameters evaluated were detected at the end of each of the three phases of the study. Concerning physiological parameters, the body weight was substantially unchanged during the three phases of the study: $424 \pm 12,432 \pm 10$, $403 \pm 7 \mathrm{~g} / \mathrm{b}$.w. respectively for phases A, B, and C. Food and water intake remained constant in each phase; the mean daily food intake value was 20.2, 23, and $19.6 \mathrm{~g}$, respectively, in phase $\mathrm{A}, \mathrm{B}$, and $\mathrm{C}$. The volume of urine produced was unchanged during phase $A$ and $B$ with a mean of $13 \mathrm{~mL}$ \pm 1 and increased slightly to $15 \mathrm{~mL} \pm 1$ during phase $\mathrm{C}$. The weight of total daily fecal production also remained constant around $7.5 \mathrm{~g} /$ day.

Concerning fecal water content, the mean water content in the feces decreased from $49.4 \pm 0.7 \%$ (phase A) to $47.1 \pm$ $0.8 \%$ after induction of constipation with loperamide (phase B). After the surgical procedure the water content of the feces increased to $51.4 \pm 1 \%$, that was significantly higher compared to the constipated rats (phase $\mathrm{C}$ versus phase $\mathrm{B}$; $P<0.0001)$. A similar trend was observed concerning the water content of the single fecal pellet, that was reduced from $59.7 \pm 0.8 \%$ (phase A) to $55.0 \pm 0.7 \%$ (phase B) and increased after the colic vein ligation to $62.6 \pm 0.9 \%$ (phase $C$ versus phase B: $P<0.0001)$.

Control Group. No significant differences were found at the end of each period in the biochemical parameters. As for the physiological parameters, the body weight was not significantly changed $(420 \pm 15,438 \pm 28,407 \pm 20$ g/b.w., resp., during phase $\mathrm{A}, \mathrm{B}$, and $\mathrm{C}$ ). Daily food intake was not different in the three phases; the daily water intake was increased in phase $\mathrm{C}$ with respect to both phases $\mathrm{A}$ and $\mathrm{B}$, but not significantly. The urine volume produced was also similar in the three phases. The daily fecal weight was increased, but not significantly in phase C: $7.5 \pm 0.5,7.1 \pm 0.4$ and $8.0 \pm 0.2 \mathrm{~g} /$ day, respectively, for phase $\mathrm{A}, \mathrm{B}$, and $\mathrm{C}$. The fecal content of water was reduced from phase A $(49.7 \pm$ $0.6 \%)$ to phase B $(46.7 \pm 0.5 \%)$. After the laxative assumption, the water content of feces increased to $48.5 \pm 0.9 \%$; (phase C versus phase $B ; P<0.0001)$. Similarly, the water content of the single fecal pellet was, respectively, $59.2 \pm 0.8,54.3 \pm 0.8$ and $58.0 \pm 0.7 \%$ in the three phases (phase $C$ versus phase $\mathrm{B} ; P<0.0001)$. No case of diarrhoea was registered both in study and control group.

3.2. Histology. We did not detect any signs of inflammation or alteration of the colic mucosa in the animals sacrificed at the different study time points, neither in the colic vein ligation group nor in the control group. However a mild mucosal edema was noticed in the animals sacrificed after ligation of the colic vein (Figure 3). The average number of PAS-positive cells detected in the crypts of colic epithelium of study group rats, was respectively, $177 \pm 2$ in phase A, $144 \pm 2$ in phase $B$ and $211 \pm 2$ cells/hpf in phase $C$ (phase $C$ versus phase B: $P<0.001)$.

In the control group, no difference was found in the histological parameters, and the average number of PAS positive cell was not significantly different in the three study phases.

\section{Discussion}

Chronic constipation is an important worldwide issue which affects almost $25 \%$ of the western population [2]. The general goal of all medical treatments for constipation is to increase fecal water content by retention of intraluminal water, increase of intestinal secretion, or shortening of intestinal transit. However in some cases these treatments are ineffective [1]. Once every medical therapeutical option has failed, surgery can be proposed as a last remedy to a selected and limited category of patients [5]. Slow transit constipation, which is unresponsive to high doses of laxatives, is considered the consequence of chronic intestinal pseudoobstruction, a rare and severe neuropathic disorder presenting with impaired gastrointestinal propulsion and bowel obstruction, without any lesion occluding the gut lumen. Patients suffering from this disease can view the surgical approach as the last option to their condition; as any surgical treatment, complication rate could be high [5]. Research for new therapeutical strategies addressing this issue is therefore strongly encouraged.

The present investigation was prompted by the observation of bowel function normalization, following arterialization of the portal vein in a patient with acute liver failure, who had previously complained of severe constipation. The report of cases of portal hypertension due to a splenic arteriovenous fistula with symptoms of diarrhea $[12,13]$ confirmed us in the concept that intestinal congestion can decrease water absorption in the colon and increase the watery content of stools, thus improving the symptoms.

In this setting we focused on evaluating the effect of the surgically induced colic vein congestion on chronic 


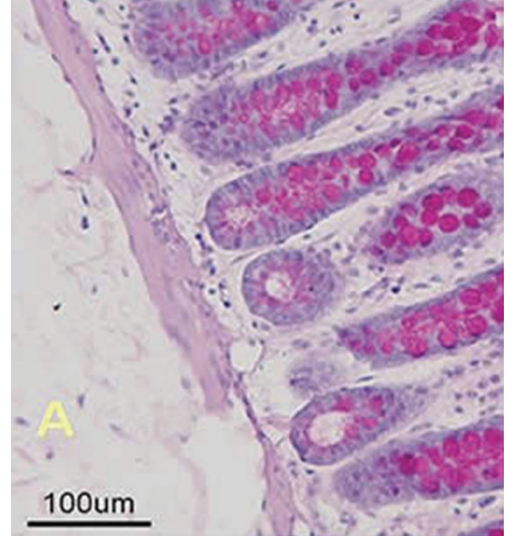

(a)

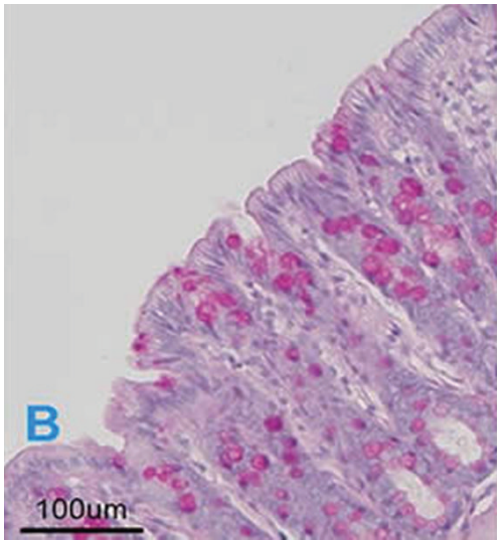

(b)

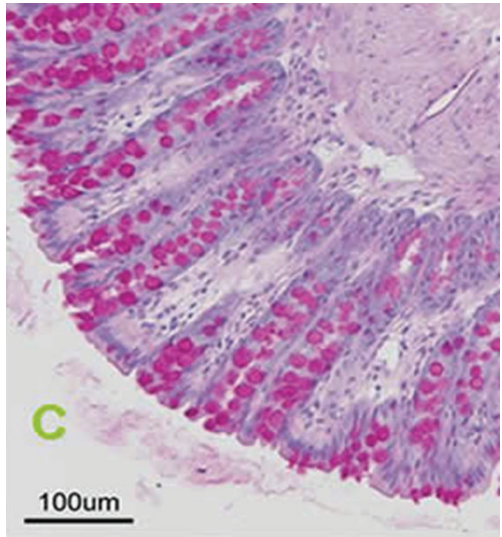

(c)

FIGURE 3: Histological images of the colic mucosa of study group animals sacrificed in the three different phases.

constipation. We employed a model of pharmacological constipation induced by loperamide (Sigma-Aldrich) [10] which can resemble the chronic functional constipation, presenting less daily fecal excretion, lower water content, and thinner mucus of fecal pellets [14]. The gastrointestinal functionality of the rats was observed in terms of quantity and water content of fecal pellets at basal condition, after loperamide injection and after surgical procedure. We did not observe a difference in the amount of feces in the three phases of the study. However, we did notice a trend of increase in water content of fecal pellets in animals treated with colic vein ligation compared to the basal and constipation phases. The results were higher than in the PEG 4,000 treatment control group, thus showing that colic vein ligation has more potent laxative effect in loperamideinduced constipation than PEG 4,000. The osmotic force for colonic fluid excretion of PEG 4,000 has already been documented [15], and the results obtained by the two different methods provide a sort of clinical validation of the present surgical model, demonstrating the "laxative like" effect of the colic vein ligation. The control group, however, did not represent a refractory constipation model, and the colic vein ligation should be preferred over the noninvasive pharmacological treatment limited to cases of medical treatment failure. Loperamide has shown to reduce the amount of mucus secreted by colic mucosa [10]; in the present investigation, we have demonstrated that the colic vein ligation can increase the amount of mucus in the colic mucosa and reverse the loperamide effect. We observed a statistically significant increase in PAS positive cells, containing mucus, in the groups of animals after colic vein ligation compared to the basal and constipated groups, suggesting that this surgical technique produces a laxative effect and alleviates the symptoms of loperamideinduced constipation. Since loperamide inhibits intestinal water secretion, as seen in human jejunum [16], it is possible that this mechanism should be compensated by the inhibition of water reabsorption in the colon, induced by the colic vein ligature with a resultant net fluid increase in the lumen.
The described procedure in rats with pharmacologically induced constipation showed to be easy and reproducible without negative apparent side effects or histologically detectable damages. These positive results prompt the development of additional experimental studies to better characterize the physiopathological mechanism beneath the observed improvement of constipation and to confirm the efficacy and safety of this surgical model in the long term and in large size animals, as swines. This step, which is crucial for the clinical translation of this research, would allow a better understanding of the effect of this procedure on a gastrointestinal system more similar to the human one. Once assessed and verified the efficacy of this therapy on other preclinical animal models, a laparoscopic approach could be applied as well, allowing to perform the procedure through minimally invasive surgery.

\section{Conclusions}

The present paper reports a tentative of surgical approach to constipation by colic vein ligation. The described procedure in rats with pharmacologically induced constipation showed to be effective, easy, and reproducible without negative apparent side effects or histologically detectable damages. These positive results prompt the development of additional experimental studies to better characterize the physiopathological mechanism beneath the observed improvement of constipation and to confirm the efficacy and safety of this surgical model in the long term and in large size animals. If these studies will confirm and fortify our preliminary results, the surgical procedure described could be considered as a valid option for selected categories of patients suffering from chronic constipation refractory to medical therapy. To this end, it is critically important that translational medical research bridges the gap between findings in basic science and data in the clinical setting.

\section{References}

[1] P. Deepak and E. D. Ehrenpreis, “Constipation,” Dis Mon, vol. 57, pp. 511-517, 2011. 
[2] P. D. R. Higgins and J. F. Johanson, "Epidemiology of constipation in North America: a systematic review," American Journal of Gastroenterology, vol. 99, no. 4, pp. 750-759, 2004.

[3] K. A. Nyrop, O. S. Palsson, R. L. Levy et al., "Costs of health care for irritable bowel syndrome, chronic constipation, functional diarrhoea and functional abdominal pain," Alimentary Pharmacology and Therapeutics, vol. 26, no. 2, pp. 237-248, 2007.

[4] G. F. Longstreth, W. G. Thompson, W. D. Chey, L. A. Houghton, F. Mearin, and R. C. Spiller, "Functional bowel disorders," Gastroenterology, vol. 130, no. 5, pp. 1480-1491, 2006.

[5] K. C. W. Hsiao, S. W. Jao, C. C. Wu, T. Y. Lee, H. J. Lai, and J. C. Kang, "Hand-assisted laparoscopic total colectomy for slow transit constipation," International Journal of Colorectal Disease, vol. 23, no. 4, pp. 419-424, 2008.

[6] American College of Gastroenterology Chronic Constipation Task Force, "An evidence-based approach to the management of chronic constipation in North America," The American Journal of Gastroenterology, vol. 100, supplement 1, pp. S1-S4, 2005.

[7] B. Nardo, R. Montalti, L. Puviani et al., "Portal vein arterialization in a patient with acute liver failure [1]," Transplantation, vol. 79, no. 7, pp. 851-852, 2005.

[8] K. Yamada and Y. Onoda, "Comparison of the effects of T1815 , yohimbine and naloxone on mouse colonic propulsion," Journal of Smooth Muscle Research, vol. 29, no. 2, pp. 47-53, 1993.

[9] Y. Sohji, K. Kawashima, and M. Shimizu, "Pharmacological studies of loperamide, an anti-diarrheal agent. II. Effects on peristalsis of the small intestine and colon in guinea pigs," Folia Pharmacologica Japonica, vol. 74, no. 1, pp. 155-163, 1978.

[10] A. Shimotoyodome, S. Meguro, T. Hase et al., "Decreased colonic mucus in rats with loperamid einduced constipation," Comparative Biochemistry and Physiology A, vol. 126, no. 2, pp. 203-212, 2000.

[11] S. Reagan-Shaw, M. Nihal, and N. Ahmad, "Dose translation from animal to human studies revisited," The FASEB Journal, vol. 22, no. 3, pp. 659-661, 2008.

[12] M. Orrego, H. E. Vargas, V. Balan et al., "Portal hypertension due to a splenic arteriovenous fistula: a case report," Digestive Diseases and Sciences, vol. 51, no. 6, pp. 1113-1116, 2006.

[13] J. H. Schmidt, R. J. Howard, M. A. Herrera, and I. F. Hawkins, "Splenic arteriovenous fistula with portal hypertension, ascites, and diarrhea," Southern Medical Journal, vol. 81, no. 5, pp. 670-672, 1988.

[14] Z. H. Yang, H. J. Yu, A. Pan et al., "Cellular mechanisms underlying the laxative effect of flavonol Naringenin on rat constipation model," PLoS One, vol. 3, no. 10, Article ID e3348, 2008.

[15] R. de Giorgio, R. Cestari, R. Corinaldesi et al., "Use of macrogol 4000 in chronic constipation," European Review for Medical and Pharmacological Sciences, vol. 15, no. 8, pp. 960 966, 2011.

[16] S. Hughes, N. B. Higgs, and L. A. Turnberg, "Loperamide has antisecretory activity in the human jejunum in vivo," Gut, vol. 25, no. 9, pp. 931-935, 1984. 


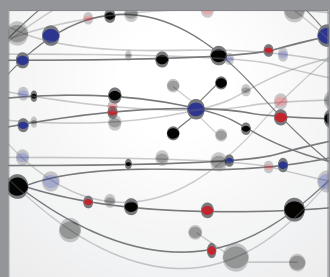

The Scientific World Journal
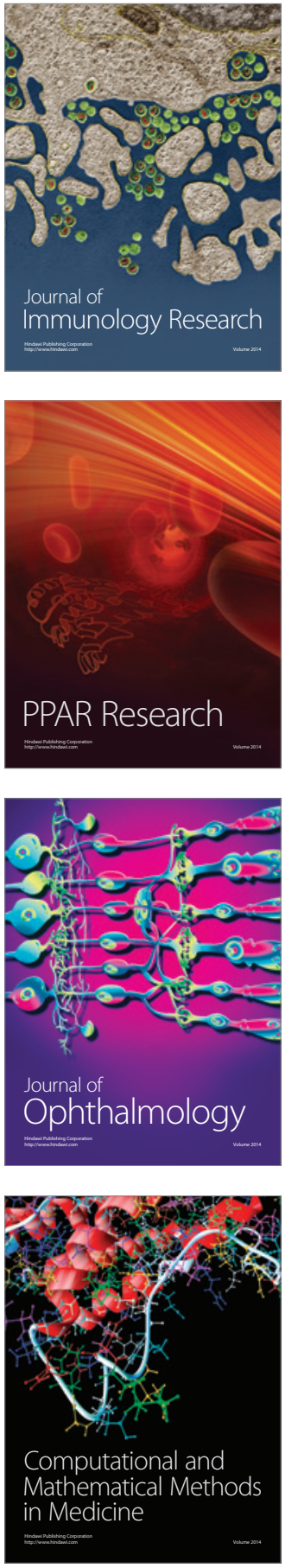

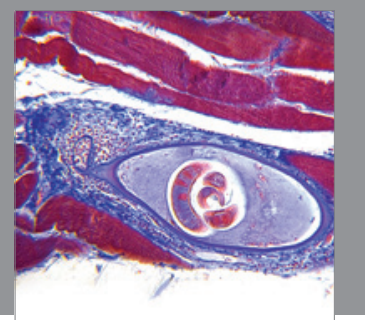

Gastroenterology

Research and Practice
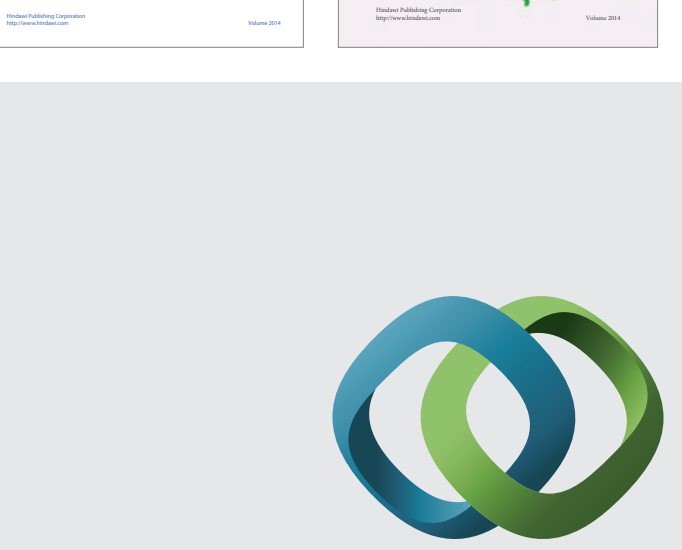

\section{Hindawi}

Submit your manuscripts at

http://www.hindawi.com
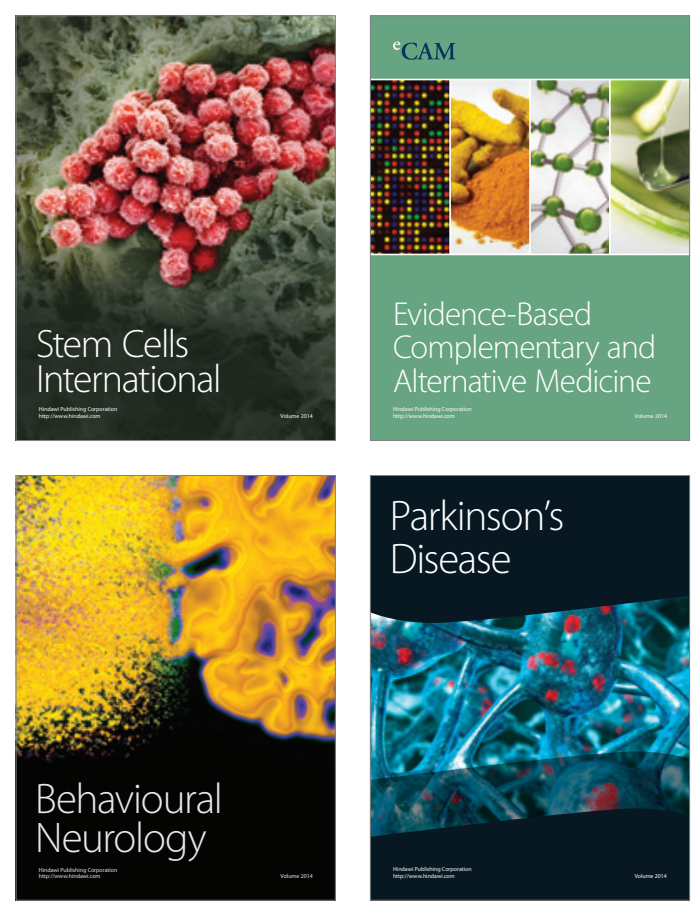

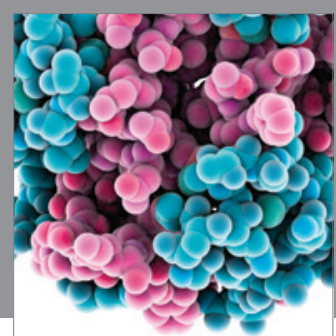

Journal of
Diabetes Research

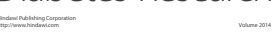

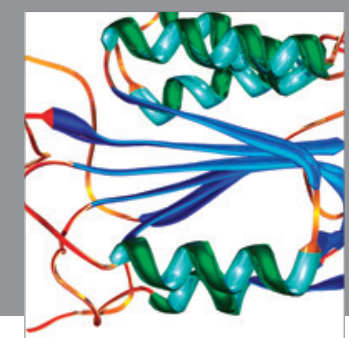

Disease Markers
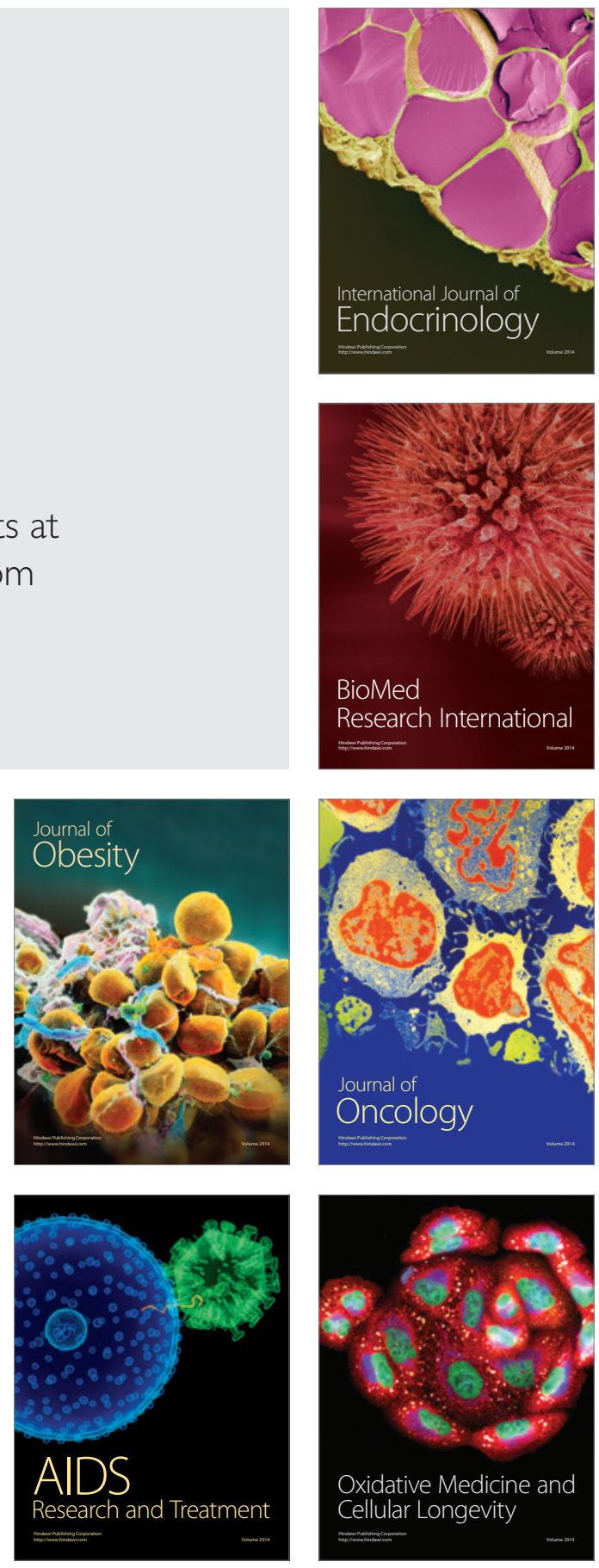